University of Wollongong

Research Online

Faculty of Engineering and Information

Faculty of Engineering and Information

Sciences - Papers: Part A

Sciences

$1-1-2014$

\title{
High-performance binary blazed grating coupler used in silicon-based hybrid photodetector integration
}

\author{
Hongqiang Li \\ Tianjin Polytechnic University \\ Yu Liu \\ Tianjin Polytechnic University, yl751@uowmail.edu.au \\ Chunjiao Miao \\ Beijing Jiaotong University \\ Meiling Zhang \\ Tianjin Polytechnic University \\ Wenqian Zhou \\ Tianjin Polytechnic University
}

See next page for additional authors

Follow this and additional works at: https://ro.uow.edu.au/eispapers

Part of the Engineering Commons, and the Science and Technology Studies Commons

Research Online is the open access institutional repository for the University of Wollongong. For further information contact the UOW Library: research-pubs@uow.edu.au 


\title{
High-performance binary blazed grating coupler used in silicon-based hybrid photodetector integration
}

\author{
Abstract \\ An efficient and high-performance binary blazed grating coupler was designed based on silicon-on- \\ insulator (SOI) used for silicon-based hybrid photodetector integration in an arrayed waveguide grating \\ demodulation integrated microsystem. A relatively high coupling efficiency was obtained to optimize \\ mode matching by the finite-difference time-domain method by choosing appropriate grating parameters, \\ including period, etching depth, and fill factor. Coupling efficiency output at $1550 \mathrm{~nm}$ for the TE mode \\ reached $68 \%$. This value was $>60 \%$ in the wavelength range of 1450 to $1600 \mathrm{~nm}$, specifically $71.4 \%$ around \\ $1478 \mathrm{~nm}$. An InP/InGaAs photodetector and SOI wafer were integrated by using benzocyclobutene (BCB) \\ bonding. When the thickness of the BCB bonding layer was $440 \mathrm{~nm}$, power absorption efficiency at 1550 \\ $\mathrm{nm}$ for the TE mode reached $78.5 \%$, whereas efficiency reached similar to $81.8 \%$ around $1475 \mathrm{~nm}$.

\section{Disciplines} \\ Engineering | Science and Technology Studies

\section{Publication Details} \\ Li, H., Liu, Y., Miao, C., Zhang, M., Zhou, W., Tang, C. \& Li, E. (2014). High-performance binary blazed grating \\ coupler used in silicon-based hybrid photodetector integration. Optical Engineering, 53 (9), \\ 097106-1-097106-6.
}

\section{Authors}

Hongqiang Li, Yu Liu, Chunjiao Miao, Meiling Zhang, Wenqian Zhou, Chunxiao Tang, and Enbang Li 


\section{Optical Engineering}

\section{High-performance binary blazed grating coupler used in silicon-based hybrid photodetector integration}

Hongqiang Li

Yu Liu

Chunjiao Miao

Meiling Zhang

Wenqian Zhou

Chunxiao Tang

Enbang Li

\section{SPIE.}




\title{
High-performance binary blazed grating coupler used in silicon-based hybrid photodetector integration
}

\author{
Hongqiang Li, ${ }^{\mathrm{a}, *}$ Yu Liu, ${ }^{\mathrm{a}}$ Chunjiao Miao, ${ }^{\mathrm{b}}$ Meiling Zhang, ${ }^{\mathrm{a}}$ Wenqian Zhou, ${ }^{\mathrm{a}}$ Chunxiao Tang, ${ }^{\mathrm{a}}$ and Enbang Li ${ }^{\mathrm{a}, \mathrm{c}}$ \\ ${ }^{a}$ Tianjin Polytechnic University, School of Electronics and Information Engineering, Tianjin 300387, China \\ beijing Jiaotong University, School of Electronics and Information Engineering, Beijing 100044, China \\ 'University of Wollongong, School of Physics, Faculty of Engineering and Information Sciences, Wollongong, NSW 2522, Australia
}

\begin{abstract}
An efficient and high-performance binary blazed grating coupler was designed based on silicon-oninsulator (SOI) used for silicon-based hybrid photodetector integration in an arrayed waveguide grating demodulation integrated microsystem. A relatively high coupling efficiency was obtained to optimize mode matching by the finite-difference time-domain method by choosing appropriate grating parameters, including period, etching depth, and fill factor. Coupling efficiency output at $1550 \mathrm{~nm}$ for the TE mode reached $68 \%$. This value was $>60 \%$ in the wavelength range of 1450 to $1600 \mathrm{~nm}$, specifically $71.4 \%$ around $1478 \mathrm{~nm}$. An $\mathrm{InP} / \mathrm{InGaAs}$ photodetector and SOI wafer were integrated by using benzocyclobutene (BCB) bonding. When the thickness of the BCB bonding layer was $440 \mathrm{~nm}$, power absorption efficiency at $1550 \mathrm{~nm}$ for the TE mode reached $78.5 \%$, whereas efficiency reached $\sim 81.8 \%$ around $1475 \mathrm{~nm}$. $\odot 2014$ Society of Photo-Optical Instrumentation Engineers (SPIE) [DOI: 10.1117/1.OE.53 .9.097106]
\end{abstract}

Keywords: silicon-on-insulator; binary blazed grating coupler; silicon-based hybrid photodetector integration.

Paper 140998 received Jun. 25, 2014; revised manuscript received Aug. 18, 2014; accepted for publication Aug. 19, 2014; published online Sep. 11, 2014.

\section{Introduction}

Photonic integrated circuits allow the implementation of multiple optical functions on a single substrate, thereby reducing the cost and size of photonic systems. These features have attracted much attention in the last decades, facilitating the development of photonic integrated circuits for telecommunication applications. Si-based optoelectronic circuits ${ }^{1}$ have attracted increasing attention because of their compact volumes and because of the high refractive index contrast of these waveguides. The large footprint and high price of the fiber Bragg grating (FBG) demodulation systems limit the promotion and application of optical FBG sensing technology. The results of our previous works on the array waveguide grating (AWG) demodulation system, which is a new kind of optical fiber grating demodulation scheme, show its suitability for optoelectronic integration. ${ }^{2}$ The system consists of an on-chip light source, multimode interface coupler, ${ }^{3}$ FBG array, $1 \times 8$ AWG, ${ }^{4}$ output grating couplers (GCs), and InP/InGaAs photodetector array as shown in Fig. 1(a). Among AWG output waveguides, GC has been used to diffract light from the silicon-on-insulator (SOI) waveguide into the photodetector with a spin-coated benzocyclobutene (BCB) layer in the middle. Figure 1(b) shows the schematic of a cross-section of the silicon-based hybrid photodetector.

Different material systems are integrated by using either wafer bonding or epitaxial growth. Due to the difficulty of epitaxial growth caused by the lattice constant difference between III-V semiconductors and silicon, wafer bonding, especially polymer bonding, appears easier. Polymer bonding photodetector on SOI wafer was realized by using a GC. Roelkens et al. ${ }^{5}$ introduced the technology for traditional GC

*Address all correspondence to: Hongqiang Li, E-mail: lihongqiang@tjpu.edu .cn and $\mathrm{InP} / \mathrm{InGaAsP}$ photodetector integration. The absorbed power fraction in the detector was $\sim 70 \%$ at a wavelength of $1500 \mathrm{~nm}$. Shao et al. ${ }^{6}$ designed a T-shaped GC used in a silicon-based hybrid photodetector integration. Power absorption efficiencies are higher, but a complex craft technique is needed. A simple process efficiently couples light in and out of the planar optical system. The binary blazed grating coupler $(\mathrm{BBGC})^{7,8}$ is a promising choice because of its novel characteristics, such as high diffraction efficiency in a specified order in an integrated optical system. However, most BBGCs reported are designed for coupling between optical fibers and SOI. As is well known, for practical application, BBGCs designed for coupling between an SOI and photodetector would be more favorable. High diffractive efficiency and easy integration of an approximately vertical out coupling structure compatible with the current fabrication facilities for CMOS technology also need to be considered.

In this study, we propose an efficient and high-performance BBGC that can be used for bonding an InP/ InGaAs photodetector onto an SOI wafer. This novel coupler includes four subperiods with different widths and fill factors, but with identical etching heights. Simulation results are obtained by using the finite-difference time-domain (FDTD) method. ${ }^{9}$ The influence of design parameters on the coupling efficiency is also discussed in detail. In silicon-based hybrid photodetector integration, high-power absorption efficiency reached $78.5 \%$ at $1550 \mathrm{~nm}$ and is $\sim 81.8 \%$ around $1475 \mathrm{~nm}$ for the TE mode and in a broad band.

\section{Device Structure and Principle}

The proposed silicon-based hybrid integrated photodetector structure is shown in Fig. 2(a), in which the core is the BBGC and is shallow-etched on the top silicon waveguide of an 


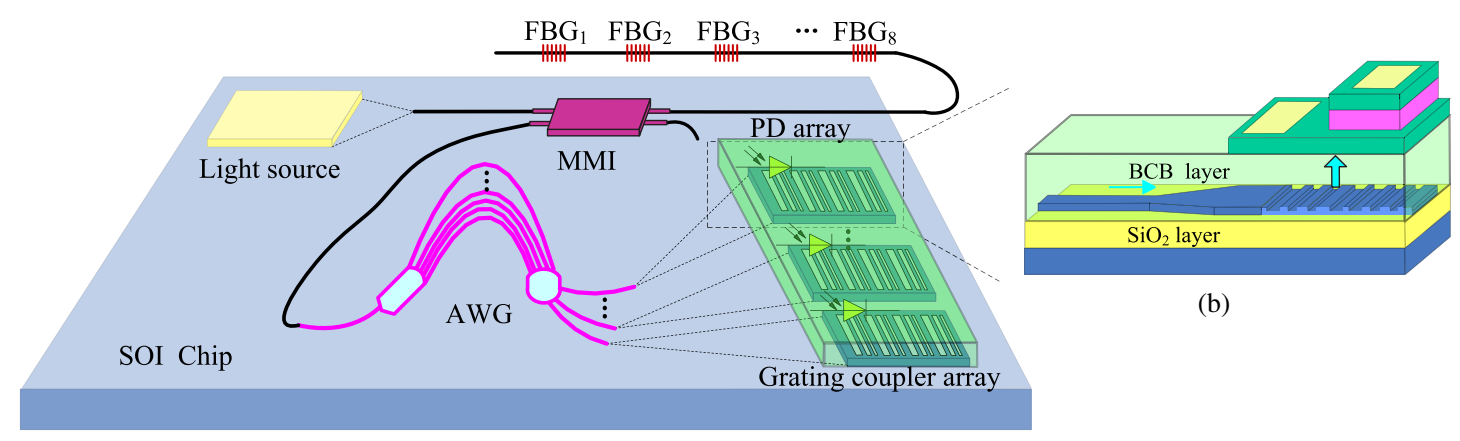

(a)

Fig. 1 (a) Array waveguide grating demodulation integrated microsystem based on silicon-on-insulator (SOI). (b) Coupling scheme of the system of the silicon-based hybrid photodetector integration.

SOI wafer. To achieve high performance, some design parameters, such as incident wavelength $\lambda$, fill factor $f$, number of pills in one period $M$, grating height $H$, grating period $T$, grating length $L, \mathrm{Si}$ layer thickness $d_{\mathrm{Si}}, \mathrm{SiO}_{2}$ buffer layer thickness $d_{\mathrm{SiO}_{2}}$, and $\mathrm{BCB}$ bonding layer thickness $d_{\mathrm{BCB}}$, are considered.

As shown in Fig. 2, the incident power $P_{\text {in }}$ is divided into four parts throughout the grating: $P_{\text {up }}, P_{\text {down }}, P_{R}$, and $P_{T}$. Coupling efficiency $\eta$ is defined as $\eta=P_{\text {up }} / P_{\text {in }}$.

According to the phase match condition between the gratings and waveguide mode, grating period $T$ should be as follows:

$T \times\left(N_{\mathrm{eff}}-n_{1} \cdot \sin \theta\right)=m \lambda \quad(\mathrm{m}=0, \pm 1, \pm 2 \ldots)$,

where $n_{1}$ is the refractive index of BCB $\left(n_{1}=1.54\right)$ and $N_{\text {eff }}$ is the effective refractive index of the waveguide for the propagation mode. Therefore, when we consider the TE mode horizontal incidence and vertical coupling, i.e., $\theta=0$, $m=1$, and $\lambda=1550 \mathrm{~nm}$, the grating period $T$ can also be acquired. When $T$ becomes long or short, the coupling efficiency has an obvious decrease due to the mode mismatch. On the basis of this original value, $T$ is further optimized by using the FDTD method. Thus, the optimum grating period is fixed at $0.7 \mu \mathrm{m}$.

Another important parameter is the fill factor. Every period of the binary grating is equally divided into $M$ subperiods with the width of $\Lambda=T / M$. The fill factor of each subperiod $f_{i}(i=1,2,3, \ldots, M)$ is defined as the ratio of the pillar width to the grating subperiod. The width of each pillar can be controlled to obtain the desired refractive index distribution. The basic design procedure and discrete

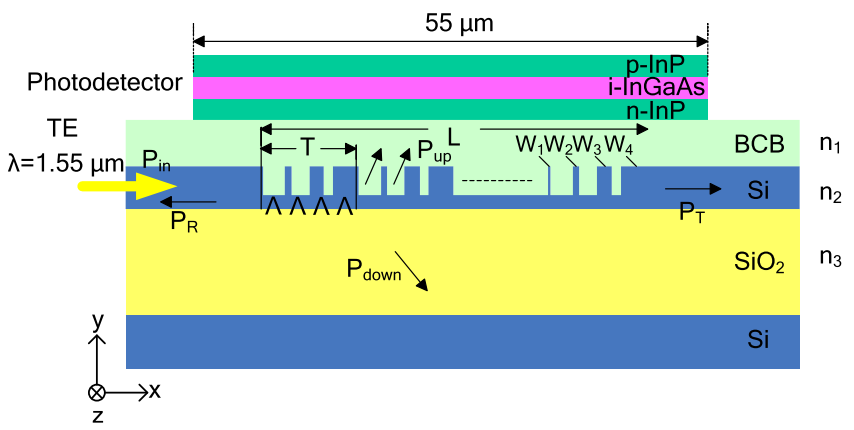

Fig. 2 Schematic structure of binary blazed grating coupler (BBGC). processing are shown in Fig. 3. We apply rigorous diffraction analysis to the localized subwavelength features within the grating period and optimize it with the simulated annealing method. ${ }^{10}$

As shown in Fig. 3, $H_{1}$ is the height of the common blazed grating. $h_{i}(i=1,2,3,4)$ is the height of each discrete multilevel grating. $H$ denotes the height of the BBGC. Then, the following equation is drawn:

$h_{i}=\frac{1}{2}\left[\frac{H_{1}}{M} \cdot i+\frac{H_{1}}{M}(i-1)\right]=\frac{(2 i-1) H_{1}}{2 M}$.

Based on the form-birefringence theory, ${ }^{11}$ the effective index of the TE mode $n_{\mathrm{eff}(i)}^{\mathrm{TE}}$ in the grating is determined by the following equation:

(a)

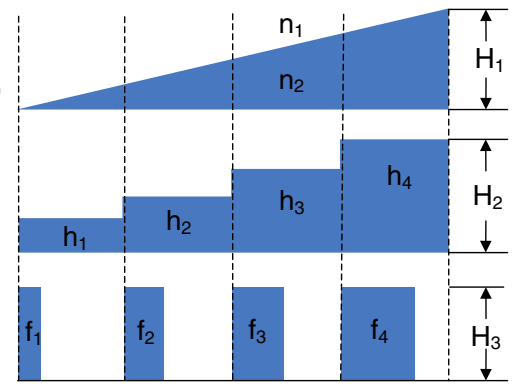

Fig. 3 Blazed grating coupler discretization process. (a) Common blazed grating. (b) Discrete multilevel grating. (c) Binary grating.

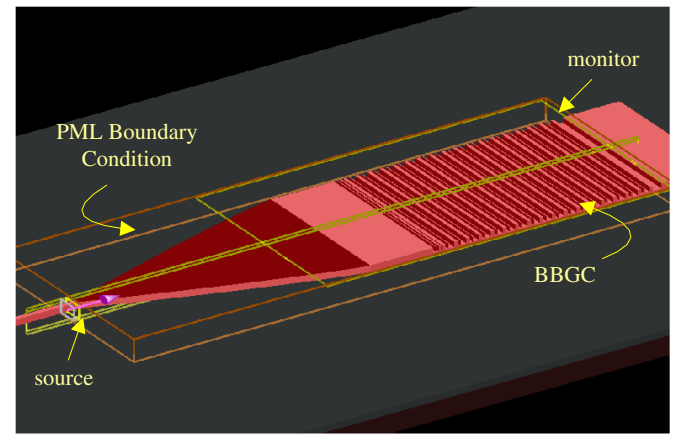

Fig. 4 BBGC structure model for three-dimensional finite-difference time-domain simulation. 


$$
n_{\mathrm{eff}(i)}^{\mathrm{TE}}=\sqrt{f_{i} n_{2}^{2}+(1-f) n_{1}^{2}}
$$

Equations (2) and (3) show that fill factors can be computed as follows:

$f_{i}=\frac{\left[\frac{2 i-1}{2 M} \frac{H_{1}}{H}\left(n_{2}-n_{1}\right)+n_{1}\right]^{2}-n_{1}^{2}}{n_{2}^{2}-n_{1}^{2}}$

$(i=1,2,3, \ldots, M)$,

where $n_{2}$ is the refractive index of $\mathrm{Si}\left(n_{2}=3.46\right)$. The above-mentioned assumptions and calculations facilitate the computation of all data required for constructing a ridgewidth-modulated grating with localized subwavelength features by using straightforward quantization of the conventional grating. Consequently, fill factors can be given when $M=4$. In our structure, considering coupling efficiency and fabrication constraint, $f_{4}$ is set to 1 , therefore, $H_{1} / H=8 / 7$ can be obtained. The remaining fill factors can be fixed $\left(f_{1}=0.096, f_{2}=0.335, f_{3}=0.636\right)$, and the width of each pillar can be acquired by $w_{i}=f_{i} \cdot \Lambda . f_{4}=1, w_{4}$ is obviously linked with $w_{1}$ in the next subperiod. Therefore, $w_{2}$ instead of $w_{1}$ is the narrowest pillar during one period, with a width of $\sim 58 \mathrm{~nm} . w_{2}$ is not difficult to fabricate using stateof-the-art lithography technology. ${ }^{12}$

For the high detector efficiency consideration of the system of silicon-based hybrid photodetector integration, the thickness of the $\mathrm{SiO}_{2}$ buffer and $\mathrm{BCB}$ bonding layers as a cavity is formed by the reflection at the $\mathrm{BCB} / \mathrm{InP}$ and $\mathrm{SiO}_{2} / \mathrm{Si}$ substrate interfaces. The thickness of silicon

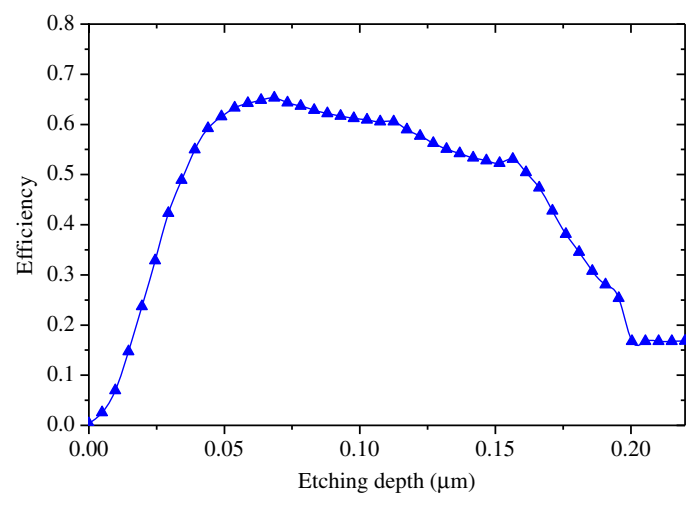

(a)

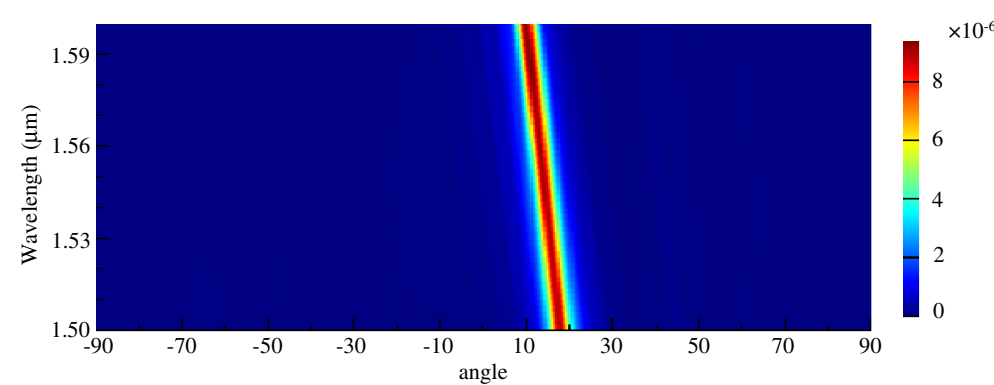

(c)

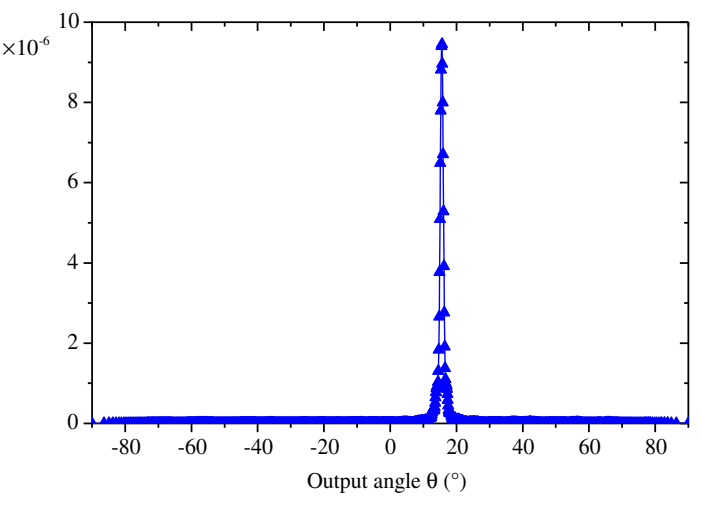

(d)

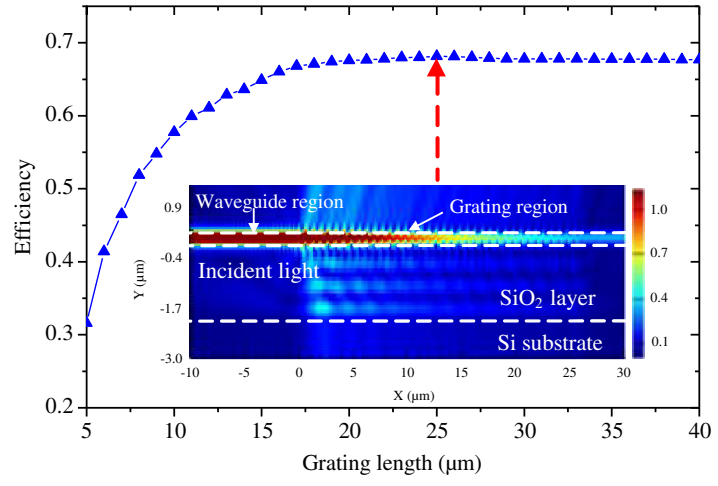

(b)

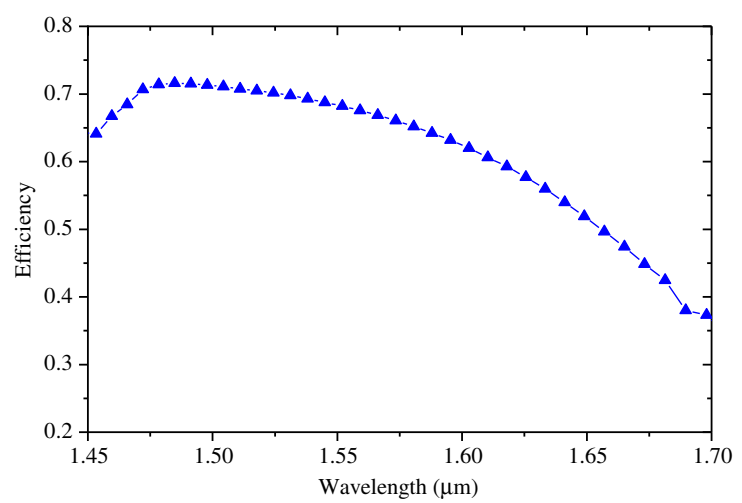

(e)

Fig. 5 (a) Relationships between output coupling efficiency and etching depth. (b) Relationships between output coupling efficiency and grating length. Inset: optical field of the output grating. (The grating length is $25 \mu \mathrm{m}$ and coupling efficiency is $\sim 68 \%$ at $\sim 1550 \mathrm{~nm}$ ). (c) The simulation of BBGC's far-field projection. (d) Output angle at $1550 \mathrm{~nm}$ wavelength. (e) Relationships between output coupling efficiency and wavelength. 
dioxide of the SOI should obey the reflection condition as follows: ${ }^{13}$

$d_{\mathrm{SiO}_{2}} \approx(2 b+1) \frac{\lambda}{4 n_{\mathrm{SiO}_{2}}}$,

where $n_{\mathrm{SiO}_{2}}$ is the refractive index of $\mathrm{SiO}_{2}\left(n_{\mathrm{SiO}_{2}}=1.45\right)$, and $b$ is an integer. The thickness of the BCB layer should act as an antireflection film to help light pass into the detector as follows: ${ }^{13}$

$$
d_{\mathrm{BCB}} \approx \frac{c \lambda}{2 n_{\mathrm{BCB}}} .
$$

\section{Analysis and Discussion}

The FDTD method is selected to simulate and design the BBGC because it is a powerful and accurate method for a finite size structure. In the simulation, a commercial FDTD software, EastFDTD (from DONGJUN TECHNOLOGY Co., Ltd., Shanghai, China) ${ }^{14}$ was used. Figure 4 illustrates the three-dimensional (3-D) model of the BBGC at TE mode light around $1550 \mathrm{~nm}$ in a $\mathrm{BCB}$ environment without a detector on top. The BBGC is modeled with boundaries that have perfectly matched layers. The total coupling efficiency obtained by 3-D FDTD simulation.

The relationship between coupling efficiency and etching depth is given in Fig. 5(a). This structure has tolerances of $110 \mathrm{~nm}$ in etched depth because the coupling efficiency is $>$ $50 \%$ when the etching depth changes from 50 to $160 \mathrm{~nm}$. Fortunately, this range is convenient for manufacturing, and due to fabrication limitation, we choose $70 \mathrm{~nm}$ as the etch depth. To improve the integration density in optical circuits, the feature sizes of the BBGC must be shrunk step by step. Overcoupling or undercoupling occurs when the grating is too long or too short, which reduces coupling efficiency accordingly. Thus, an optimum grating length must be chosen. The relationships between the grating length and efficiency are shown in Fig. 5(b). As shown in Fig. 5(b), the coupling efficiency first increases monotonously with the grating length and then only slightly changes with the grating length. Moreover, the photosensitive area of the photoelectric detector used in this study is a circle with a diameter of $55 \mu \mathrm{m}$. Thus, a grating length of $25 \mu \mathrm{m}$ is chosen. The optical field of the output grating calculated by the FDTD method is shown in the inset of Fig. 5(b). At a wavelength of 1500 to $1600 \mathrm{~nm}$, the peak angle is between 11.4 and $20.5 \mathrm{deg}$ as shown in Fig. 5(c), which is obtained from the monitor's far-field projection. Far-field projection shows $\sim 15.6 \mathrm{deg}$ emission at $1550 \mathrm{~nm}$, as depicted in Fig. 5(d). The output coupling efficiency at $1550 \mathrm{~nm}$ for the TE mode reached $68 \%$, which is $>60 \%$ in the wavelength range of 1450 to $1600 \mathrm{~nm}$, and is $71.4 \%$ around $1478 \mathrm{~nm}$, as shown in Fig. 5(e). All important parameters used in the simulation are given in Table 1.

The tapered part of the BBGC was simulated with the beam propagation method (BPM). The length of the taper required to create a transition with a negligible loss between the 450-nm-wide silicon wire waveguide and grating $(W=10.8 \mu \mathrm{m})$ was $300 \mu \mathrm{m}$, determined through linear tapering. Figure 6 shows the input waveguide with the tapered part in the BPM simulation region. Figure 6(a) displays the height-coded graph that shows the distribution of the electric field intensity along the propagation direction in the tapered part and input waveguide. The input mode is smoothly distributed across the cross-section. Figure 6(b) shows the contour map ( $x-z$ plane), $85 \%$ of which is from the input optical power. The optical field from BPM

Table 1 Design parameters of binary blazed grating coupler (BBGC).

\begin{tabular}{llccc}
\hline Grating period & Etching depth & Si layer thickness & Grating length & Pillars in one period \\
\hline$T=0.7 \mu \mathrm{m}$ & $H=0.07 \mu \mathrm{m}$ & $d_{\mathrm{Si}}=0.22 \mu \mathrm{m}$ & $L=25 \mu \mathrm{m}$ & $M=4 \quad \begin{array}{c}f_{1}=0.096, f_{2}=0.335, \\
f_{3}=0.636, f_{4}=1\end{array}$ \\
\hline
\end{tabular}

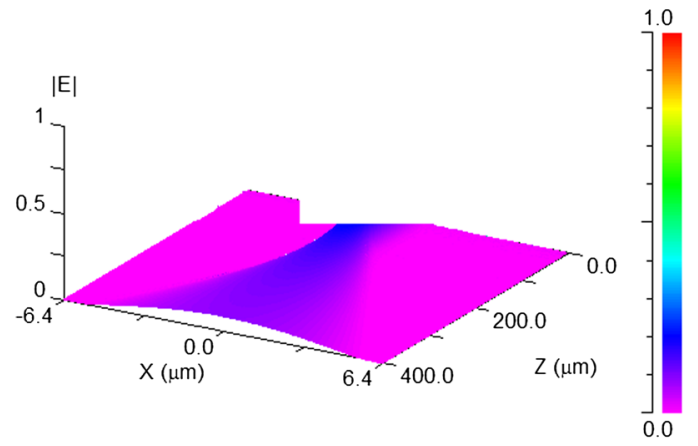

(a)

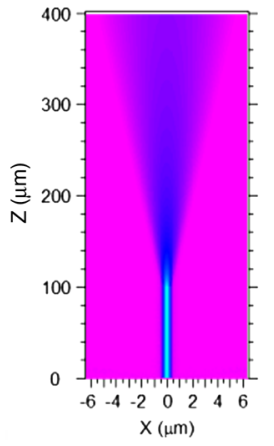

(b)

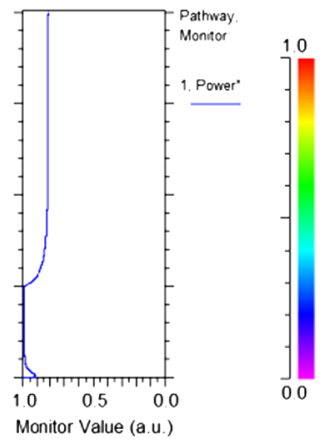

Fig. 6 Input waveguide and tapered part of BBGC in beam propagation method simulation region. (a) Height-coded intensity of electrical field. (b) Contour map ( $x-z$ plane) with optical power along propagation direction. 


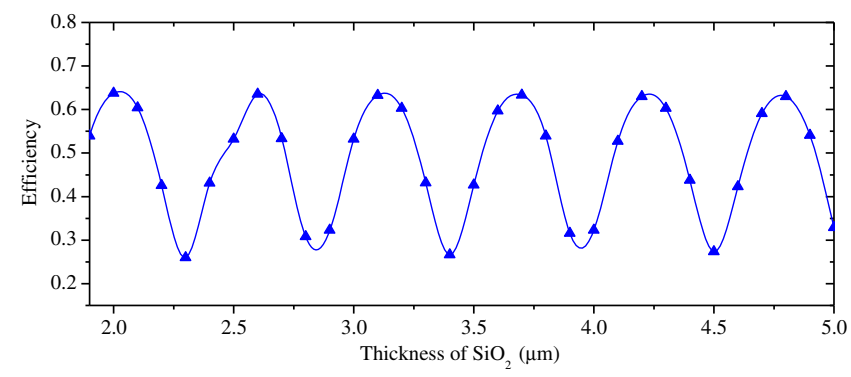

Fig. 7 Relationships between the output coupling efficiency and the thickness of $\mathrm{SiO}_{2}$ buffer layer.

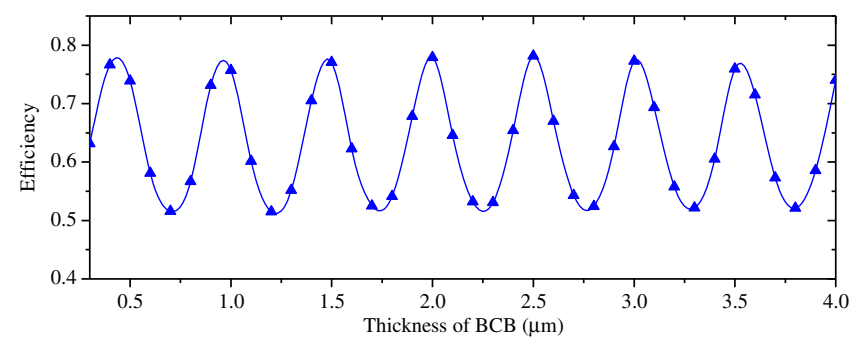

Fig. 8 Relationships between the power absorption efficiency and the thickness of benzocyclobutene bonding layer.

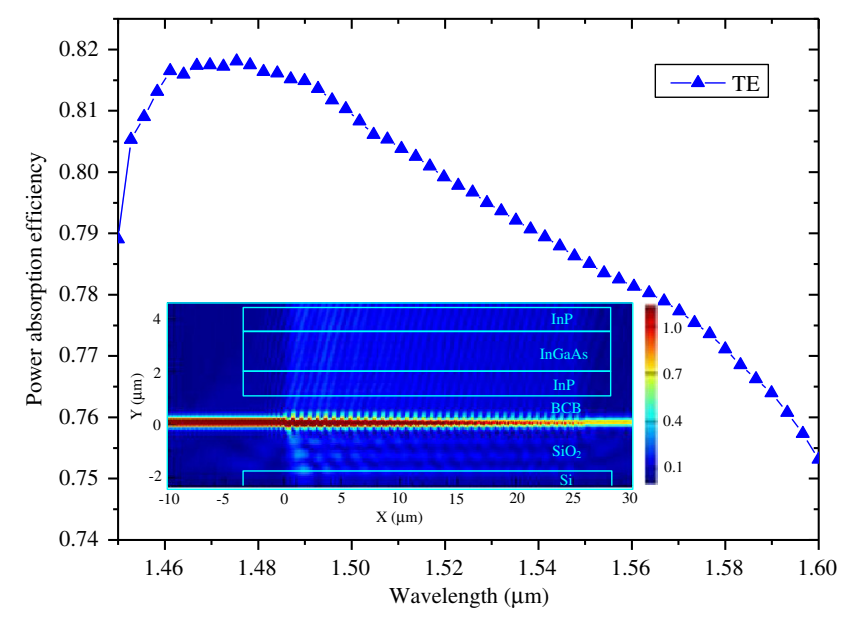

Fig. 9 Relationships between power absorption efficiency and wavelength. Inset: simulation of light diffraction from the SOI waveguide toward the $\mathrm{InP} / \mathrm{InGaAs}$ photodetector at $1550 \mathrm{~nm}$. simulation at the end of taper was used as the input field of the BBGC of the FDTD simulation tool.

An InP/InGaAs photodetector is bonded onto this BBGC with a spin-coated BCB layer in the middle to achieve Sibased hybrid photodetector integration. First, the $\mathrm{SiO}_{2}$ buffer layer thickness is optimized in a BCB environment without a detector on top. Relationships between the thickness of the $\mathrm{SiO}_{2}$ buffer layer and the output coupling efficiency for the TE mode are shown in Fig. 7. Specifically, the buffer layer and coupling efficiency oscillate because of reflection and antireflection effects. The thickness of the $\mathrm{SiO}_{2}$ buffer layer is chosen as $2 \mu \mathrm{m}$, which accords with the whole arrayed waveguide grating demodulation system with a standard SOI chip.

Then, the thickness of the BCB layer is optimized to determine how much influence the thickness has on the power absorption efficiency of the InP/InGaAs photodetector. The thicknesses of the top InP layer and the lower InP layer are assumed to be $1 \mu \mathrm{m}$, and the thickness of the InGaAs absorbing layer is $2 \mu \mathrm{m}$. Relationships between the thickness of the BCB bonding layer and power absorption efficiency for the TE mode are shown in Fig. 8.

The bonding layer and power absorption efficiency also oscillate because of reflection and antireflection effects. The thickness of the BCB bonding layer is $440 \mathrm{~nm}$. Relationships between wavelength and power absorption efficiency for the TE mode are shown in Fig. 9. Simulation results of light diffraction at $1550 \mathrm{~nm}$ for the TE mode are shown in the inset of Fig. 8; these results indicate high power absorption efficiencies. A power absorption efficiency of $78.5 \%$ is achieved at $1550 \mathrm{~nm}$ and is $\sim 81.8 \%$ around $1475 \mathrm{~nm}$. Power absorption efficiencies $>75 \%$ are realized in a wide wavelength range of 1450 to $1600 \mathrm{~nm}$. Table 2 shows a comparison of grating couplers designed in recent years.

\section{Conclusion}

This study proposes an efficient and high-performance BBGC used for silicon-based hybrid photodetector integration in an AWG demodulation integrated microsystem. The grating region was $10.8 \mu \mathrm{m}$ wide and $25 \mu \mathrm{m}$ long. High power absorption efficiency of the InP/InGaAs photodetector for the TE mode is acquired in a broad band. Power absorption efficiency at $1550 \mathrm{~nm}$ for the TE mode reached $78.5 \%$ and was $>75 \%$ in a wide wavelength range of 1450 to $1600 \mathrm{~nm}$. Notably, an $81.8 \%$ power absorption efficiency was achieved around $1475 \mathrm{~nm}$. Simulation results and theoretical analysis show that the BBGC is useful for integrated optical circuits. Furthermore, it is not difficult to fabricate

Table 2 Comparison of grating couplers.

\begin{tabular}{lccr}
\hline Reference (year) & Grating coupler types & Coupling application & Efficiency \\
\hline $15(2009)$ & Fully etched grating coupler & Fiber to waveguide & $49 \%-1.55 \mu \mathrm{m}$ \\
$16(2010)$ & Apodized grating coupler & Fiber to chip & $43 \%-1.53 \mu \mathrm{m}$ \\
$17(2011)$ & Multilevel grating coupler & Waveguide to waveguide & $67.5 \%-1.45 \mu \mathrm{m}$ \\
$18(2013)$ & Vertical grating coupler & Fiber to waveguide & $37 \%-1.55 \mu \mathrm{m}$ \\
Present paper (2014) & BBGC & Waveguide to photodetector & $78.5 \%-1.55 \mu \mathrm{m}$ \\
\hline
\end{tabular}


this grating with conventional CMOS technology, which will be useful for integrated optical circuits. The designed BBGC is a promising material for the silicon-based hybrid integration of photodetectors because of its high efficiency and compact structure.

\section{Acknowledgments}

This work was supported by the National Natural Science Foundation of China (Nos. 61177078, 61307094, 31271871), the Specialized Research Fund for the Doctoral Program of Higher Education of China (No. 20101201120001), and Tianjin Research Program of Application Foundation and Advanced Technology (No. 13JCYBJC16800).

\section{References}

1. B. Jalali and S. J. Fathpour, "Silicon photonics," J. Lightwave Technol. 24(12), 4600-4615 (2006).

2. H. Li et al., "Preliminary investigation of an SOI-based arrayed waveguide grating demodulation integration microsystem," Sci. Rep. 4(4848), 1-6 (2014).

3. H. Li et al., "Design optimization and comparative analysis of siliconnanowire-based couplers," IEEE Photonics J. 4(5), 2017-2026 (2012).

4. $\mathrm{H}$. Li et al., "Design of $1 \times 8$ silicon nanowire arrayed waveguide grating for on-chip arrayed waveguide grating demodulation integration microsystem," Opt. Eng. 51(12), 123001 (2012).

5. G. Roelkens et al., "Integration of InP/InGaAsP photodetectors onto silicon-on-insulator waveguide circuits," Opt. Express 13(25), 10102-10108 (2005).

6. S. Shao et al., "Design of a T-shaped grating coupler used in siliconbased hybrid photodetector integration," in 7th IEEE Int. Conf. on Group IV Photonics, pp. 132-134, IEEE (2010).

7. J. Yang et al., "High efficient subwavelength binary blazed grating beam splitter via vertical coupling," IEEE Photon. Technol. Lett. 23(13), 896-898 (2011).

8. J. Yang et al., "High-performance and compact binary blazed grating coupler based on an asymmetric subgrating structure and vertical coupling," Opt. Lett. 36(14), 2614-2617 (2011).

9. A. Taflove and S. C. Hagness, Computationai Electrodynamics, Artech House, London, United Kingdom and Boston (2000).

10. Z. Zhou and T. J. Drabik, "Optimized binary, phase-only, diffractive optical element with subwavelength features for $1.55 \mathrm{~mm}$," J. Opt. Soc. Am. A 12(5), 1104-1112 (1995)
11. N. Lu, D. Kuang, and G. Mu, "Design of transmission blazed binary gratings for optical limiting with the form-birefringence theory," Appl. Opt. 47(21), 3743-3750 (2008).

12. J. Bolten et al., "CMOS compatible cost-efficient fabrication of SOI grating couplers," J. Microelectron. Eng. 86(4-6), 1114-1116 (2009).

13. J. Mu, H. Zhang, and W. Huang, "Design of waveguide Bragg gratings with strong index corrugations," J. Lightwave Technol. 26(12), 1596-1601 (2008)

14. DONGJUN TECHNOLOGY Co., Ltd. China "EastFDTD v4.0 Beta," http://www.eastfdtd.com (2013).

15. B. Schmid, A. Petrov, and M. Eich, "Optimized grating coupler with fully etched slots," Opt. Express 17(13), 11066-11076 (2009).

16. R. Halir et al., "Continuously apodized fiber-to-chip surface grating coupler with refractive index engineered subwavelength structure," Opt. Lett. 35(19), 3243-3245 (2010).

17. W. Zhou et al., "Ultrabroad bandwidth and high-coupling-efficiency compact coupler using multilevel grating structure," Proc. SPIE 8191, 819120 (2011).

18. Z. Zhang et al., "CMOS-compatible vertical grating coupler with quasi Mach-Zehnder characteristics," IEEE Photon. Technol. Lett. 25(3), 224-227 (2013).

Hongqiang Li received his $\mathrm{BE}, \mathrm{MSc}$, and $\mathrm{PhD}$ degrees from Tianjin Polytechnic University. He is a professor in the School of Electronics and Information Engineering, Tianjin Polytechnic University. His research interests lie in the area of optical fiber grating sensor demodulation and optoelectronics integration.

Yu Liu is working on her master's degree in the School of Electronics and Information Engineering, Tianjin Polytechnic University.

Chunjiao Miao is working on her doctor's degree in the School of Electronics and Information Engineering, Beijing Jiaotong University.

Meiling Zhang is a vice professor in the School of Textile Division, Tianjin Polytechnic University.

Wenqian Zhou is working on his master's degree in the School of Electronics and Information Engineering, Tianjin Polytechnic University.

Chunxiao Tang is a lecturer in the School of Electronics and Information Engineering, Tianjin Polytechnic University.

Enbang Li is a professor in the School of Physics, Faculty of Engineering and Information Sciences, University of Wollongong. 